\title{
Perforant Pathway
}

National Cancer Institute

\section{Source}

National Cancer Institute. Perforant Pathway. NCI Thesaurus. Code C13084.

The route of neural fibers originating in the entorhinal cortex that project through the hippocampal formation to granule cells of the dentate gyrus, providing the major input to the hippocampus. 